\title{
An InGrid based Low Energy X-ray Detector for the CAST Experiment
}

\author{
Christoph Krieger ${ }^{*}{ }^{a}$ Klaus Desch, ${ }^{a}$ Jochen Kaminski, ${ }^{a}$ Michael Lupberger ${ }^{a}$ and \\ Theodoros Vafeiadis ${ }^{b}$ \\ ${ }^{a}$ University of Bonn, Germany \\ ${ }^{b} C E R N$ \\ E-mail: krieger@physik.uni-bonn.de
}

The CERN Axion Solar Telescope (CAST) is searching for axions and other new particles like chameleons coupling to photons and emerging from the Sun. Those particles are converted into soft X-ray photons in a high magnetic field. To enhance sensitivity for physics beyond the Standard Model it is necessary to cope with weak couplings and low energies, thus requiring an efficient background discrimination as well as a detection threshold below $1 \mathrm{keV}$.

A promising candidate for a future CAST detector is an InGrid based X-ray detector. This detector combines the high spatial resolution of a pixelized readout with a highly granular Micromegas gas amplification stage. Fabrication by photolithographic postprocessing techniques allows to match the amplification grid to the pixels. The high granularity facilitates the detection of single electrons which allows to determine the X-ray energy by electron counting. Additionally, rejection of background events mostly originating from cosmic rays is provided by an event shape analysis exploiting the high spatial resolution.

In order to demonstrate its low detection threshold, an InGrid based detector was tested in the CAST Detector Lab where an X-ray generator for energies down to a few hundred eV is available. Results of these tests demonstrate the detector's ability to detect the carbon K-alpha line at 277 $\mathrm{eV}$. The detector was mounted afterwards at the CAST experiment behind an X-ray telescope in order to take data during the 2014 run of CAST.

Technology and Instrumentation in Particle Physics 2014

2-6 June, 2014

Amsterdam, the Netherlands

\footnotetext{
${ }^{*}$ Speaker.
} 


\section{Axions \& Chameleons}

As the latest high precision measurement of the cosmic microwave background performed by the Planck satellite implies [1], roughly $95 \%$ of the energy in the Universe is of a form which up to now could not be detected or identified directly. This unknown form of energy splits up into two parts: Dark Matter (26.8\%) and Dark Energy (68.3\%). A possible Dark Matter candidate can be found in the solution to the strong CP-problem.

The strong $\mathrm{CP}$-problem is the non-observation of $\mathrm{CP}$ violation in strong interactions although it is not forbidden by theory. It is equivalent with the non-existence of an electric dipole moment of the neutron which is following the most precise measurement excluded up to a value of $0.29 \times 10^{-25} e \mathrm{~cm}$ [2]. The strong CP-problem is cured by the Peccei-Quinn mechanism which gives rise to a Pseudo-Goldstone-Boson, the axion, at the point at which symmetry breaking is involved [3, 4].

Through mixing with neutral pions axions acquire a small mass, their couplings to matter are however very small. This and their long lifetime make them an ideal candidate for at least a fraction of Dark Matter. Due to the mixing with neutral pions they also have a coupling to two photons. As one of these photons can be virtual, axions can be produced in strong electromagnetic fields by converting real photons into axions; this is called the Primakoff effect.

The Chameleon however can be one of the very few candidates for what is called Dark Energy. In this sense what is observed as Dark Energy is explained by the existence of scalar fields with couplings to matter as well as to photons. To avoid a fifth force with long range and large gravitational effects the chameleon screening mechanism can be exploited [5] which results in a model with an effective mass which depends on the density of the surrounding medium. Due to the photon coupling chameleons can be converted to photons in strong electromagnetic fields and vice versa, like axions.

\section{The CERN Axion Solar Telescope}

The CERN Axion Solar Telescope (CAST) searches for axions produced in the Sun [6]. Through the Primakoff effect in the Sun's core a high axion flux is produced which reaches the Earth. To detect these solar axions a high magnetic field can be exploited to convert them back into photons by the inverse Primakoff effect. Due to energy conservation the energy distribution of these photons resembles the energy distribution of the photons in the Sun's core and therefore the temperature at the core. Back-conversion of solar axions produces photons of up to $15 \mathrm{keV}$ with the flux peaking at $\sim 3 \mathrm{keV}$. However, the rate of photons is rather low due to the weak axion couplings, so that detectors with low background rates are required.

CAST uses a decommissioned LHC prototype magnet to generate the high magnetic field needed. The magnet can be pointed to the Sun for $1.5 \mathrm{~h}$ during sunrise and sunset. The remaining time of the day is used to take background data. On both ends of the magnet X-ray detectors are mounted. Three out of four stations are equipped with microbulk Micromegas detectors and until mid of 2013 an X-ray telescope in combination with a pnCCD detector [7] was used at the fourth station. 
Although up to now no axions could be found, CAST could set the most stringent limits on solar axions in the mass range this kind of experiment is sensitive to [8]. Future plans for the CAST experiment include further improvement of the detectors which means to reduce the background rates to further improve the experiment's sensitivity. But also an additional search for solar chameleons is planned. In contrast to axions solar chameleons are produced not in the Sun's core but in a small shell close to the tachocline region. Due to lower temperature at this region chameleons are produced with less energy which results in the flux peaking dependent on the assumed model and production mechanism below or slightly above $1 \mathrm{keV}$ [5]. To enable the chameleon search at CAST low energy X-ray detectors with a threshold below $1 \mathrm{keV}$ and low enough background rates are needed.

\section{An InGrid based Low Energy X-ray Detector}

A possible way to lower the energy threshold of Micromegas detectors is to increase the granularity of their readout plane so that individual electrons from the X-ray photon conversion in the gaseous medium can be detected. To accommodate for the high density of channels it is more suitable to use integrated readout electronics in the form of a pixel chip in combination with a Micromegas gas amplification stage instead of a conventional pad based readout scheme. This can be achieved by producing a Micromegas like stage on top of a pixel chip by means of photolithographic postprocessing techniques [9, 10]. This structure (see figure 1a) is called an InGrid.

The pixel chip used is the Timepix ASIC [11] (see figure 1b). This is a pixel chip with $256 \times 256$ pixels with a pixel pitch of $55 \mu \mathrm{m}$ and an overall active area of $1.4 \times 1.4 \mathrm{~cm}^{2}$. Each of the pixels provides a charge sensitive amplifier and a single threshold discriminator and allows to measure either charge (time over threshold) or time (time of arrival). The time measurement however requires an external trigger signal as the integrated counters do not provide appropriate overflow features. The readout mode of the Timepix ASIC is frame based. The electronics need to be protected from discharges in the gas amplification stage, therefore a resistive layer made of $4 \mu \mathrm{m}$ silicon nitride which spreads the charge in case of a spark [12] is deposited on the chip before. As readout system an FPGA-based system developed at the University of Bonn is used for the InGrid based X-ray detector [13].

The InGrid based low energy X-ray detector uses the Timepix ASIC with an InGrid stage as central detection device. The detector design (see figure 2 for CAD drawings) is based on the design of the CAST Micromegas detectors [14] and features a body made of acrylic glass and a modular readout housing Timepix ASIC and InGrid stage on a small carrier board. The chip is covered by a field shaping electrode with a cutout just the size of the chip's active area which rests slightly above the InGrid's mesh and is put to the appropriate electrical potential in order to reduce field distortions arising at the chip borders and especially at the bond area where the chip is connected to the underlying boards. The drift distance available between the anode and cathode of the detector is $3 \mathrm{~cm}$ and a drift field of $500 \mathrm{~V} \mathrm{~cm}^{-1}$ is applied. The cathode frame is made of copper with a thin film glued on, which acts as entrance window for X-ray photons. To allow very low energetic photons to enter the detector the copper was removed in a 16 by $16 \mathrm{~mm}^{2}$ area. Only a support grid-like structure was kept and a $2 \mu \mathrm{m}$ thick metalized Mylar film was glued on it. This configuration withstands a pressure difference of $1050 \mathrm{mbar}$, important to connect the gas-filled 


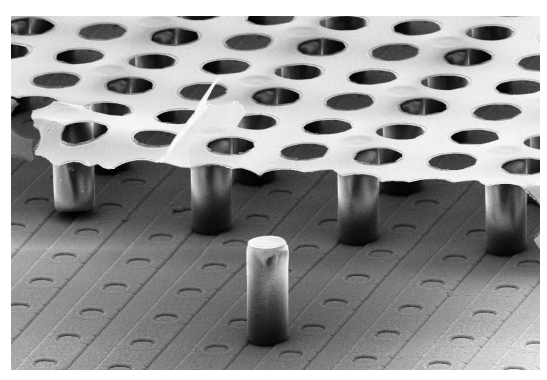

(a)

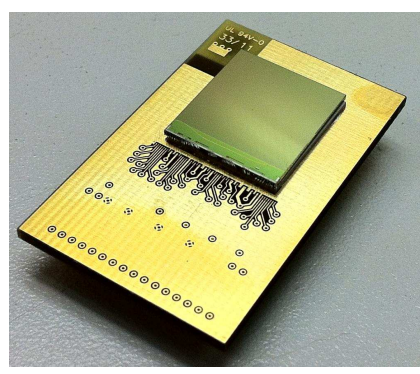

(b)

Figure 1: SEM image of an InGrid structure on top of a Timepix ASIC (a), taken from [13]. In the SEM image parts of the mesh have been removed to show the good alignment between pixels and mesh holes. And a bare Timepix ASIC on a carrier board (b).

detector to a vacuum beam pipe. Mylar of $2 \mu \mathrm{m}$ thickness has been chosen as a good compromise between transparency (see figure $3 \mathrm{a}$ ) and robustness.

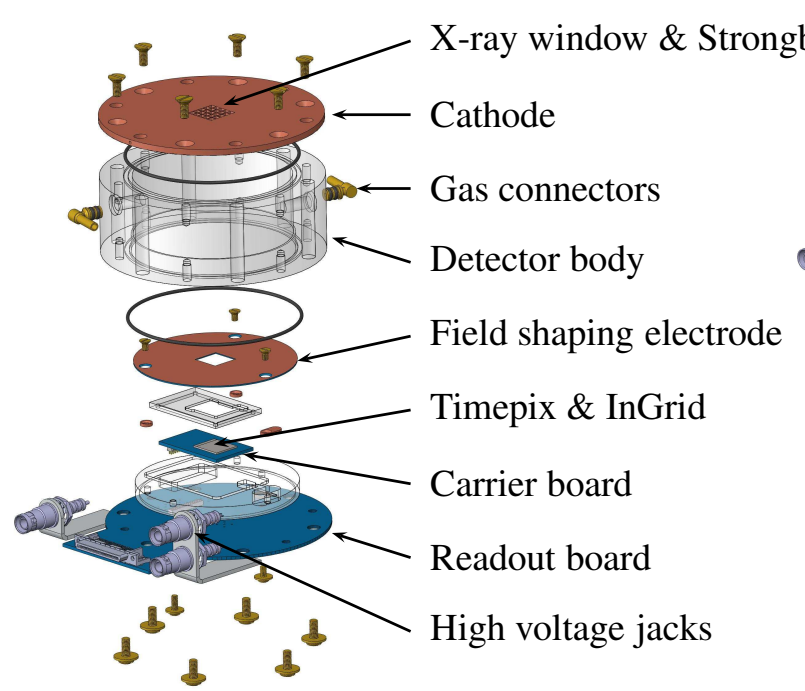

(a)

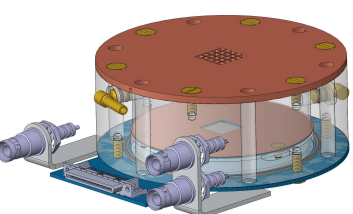

(b)

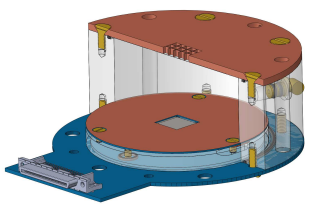

(c)

Figure 2: CAD drawings of the InGrid based X-ray detector. An exploded view is shown in (a) including labeling of main parts. The assembled detector is depicted in (b) and a view to the inside showing the Timepix and InGrid embedded in the field shaping electrode at the center of the detector is shown in (c).

$\mathrm{X}$-ray photons entering the detector will likely hit a gas atom and produce via ionization a bunch of primary electrons which will drift towards the readout plane. Due to diffusion they will be collected as an electron cloud of circular shape on the readout chip. As the individual electrons of this cloud are detected, the detector features a low threshold together with a topological background suppression by application of an event-shape analysis. A sample X-ray event is shown in comparison to a track event in figure 4. 


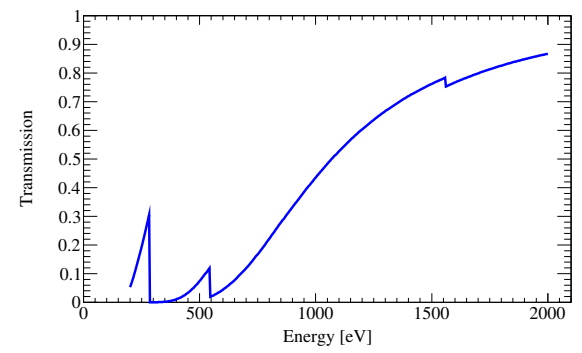

(a)

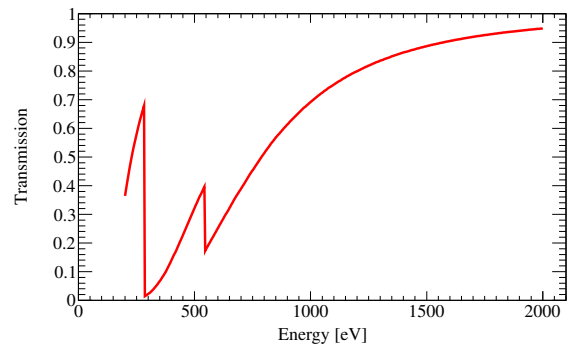

(b)

Figure 3: Transmission curves for thin Mylar films in the energy range up to $2 \mathrm{keV}$. $2 \mu \mathrm{m}$ thick Mylar metalized with $40 \mathrm{~nm}$ of aluminum (a) as used for detector's entrance window and $0.9 \mu \mathrm{m}$ thick Mylar (b) as used for the differential window. Transmission data was taken from [15].

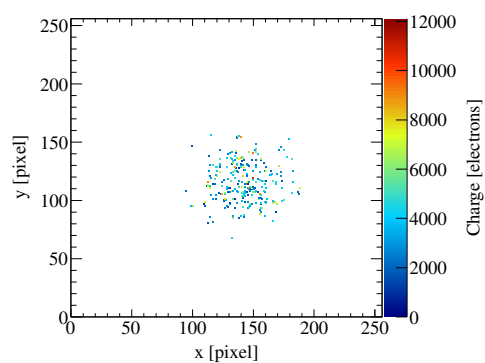

(a)

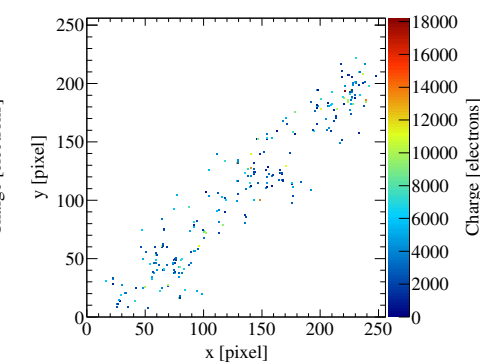

(b)

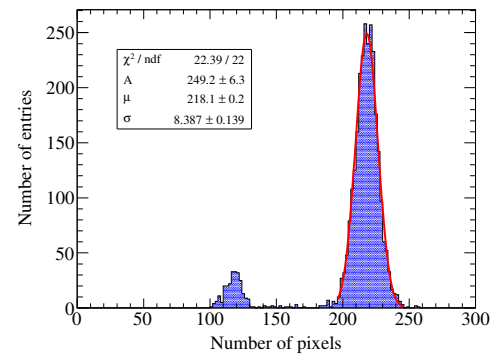

(c)

Figure 4: Event displays showing a typical X-ray event of $5.9 \mathrm{keV}$ (a) and a track produced by a cosmic ray traversing the detector (b). The area depicted is the complete active area of the Timepix ASIC. $\mathrm{Z}$ axis displays the charge collected on each pixel. Figure (c) shows a spectrum recorded with an ${ }^{55} \mathrm{Fe}$ source and a gas mixture of Argon and isobutane with $10 \%$ quencher fraction. A Gaussian function has been fitted to the photopeak at $5.9 \mathrm{keV}$ revealing an energy resolution of $\sigma_{E} / E=3.85 \%$.

By applying a background suppression method based on a likelihood ratio scheme using reference data sets, a first prototype was able to achieve background rates in the order of a few times $10^{-5} \mathrm{~cm}^{-2} \mathrm{~s}^{-1} \mathrm{keV}^{-1}$ [16]. Additionally energy resolutions $\sigma_{E} / E$ of down to $3.85 \%$ at $5.9 \mathrm{keV}$ could be achieved at optimized settings in a gas mixture of Argon and isobutane with $10 \%$ quencher fraction and energy determination by simple pixel (or electron) counting. This value, however, is already close to the lower limit of roughly $3 \%$ given by the Fano factor [17]. The corresponding spectrum is shown in figure $4 \mathrm{c}$, again the number of fired-pixels is taken as measure of the photon energy. For safety reasons a mixture with only $2.3 \%$ isobutane will be used at the CAST experiment. This mixture still gives an energy resolution of $5.3 \%$, the decrease of performance is due to nonlinear effects in the gas amplification process partly correlated with the increased range of UV photons which comes along with the lower fraction of quenching gas. 


\section{Tests at the X-ray Generator of the CAST Detector Lab}

In order to prove the low detection threshold of the InGrid based detector, tests at the detector lab of the CAST collaboration have been carried out. In this lab an X-ray generator is available which features a set of exchangeable targets as well as a set of dedicated filters to obtain clean $\mathrm{X}$-ray spectra with single lines [18]. Additionally, a complete vacuum beamline including the infrastructure for differential pumping is available. The differential pumping is needed to compensate for the gas permeation through the thin window in order to still achieve a reasonable good vacuum to operate the X-ray tube. As differential window a $0.9 \mu \mathrm{m}$ thick Mylar film (see figure $3 \mathrm{~b}$ for its transmission curve) was used. Figure 5 shows pictures of the X-ray generator and the InGrid based detector mounted to the beamline.

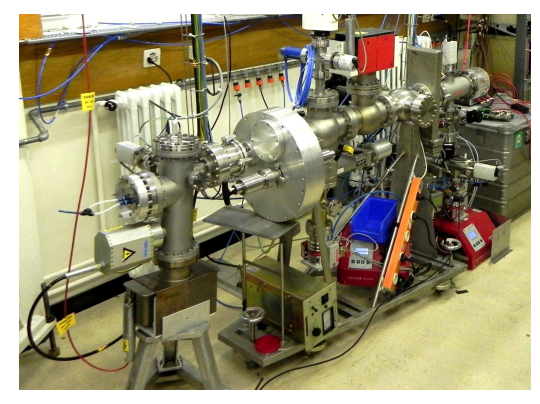

(a)

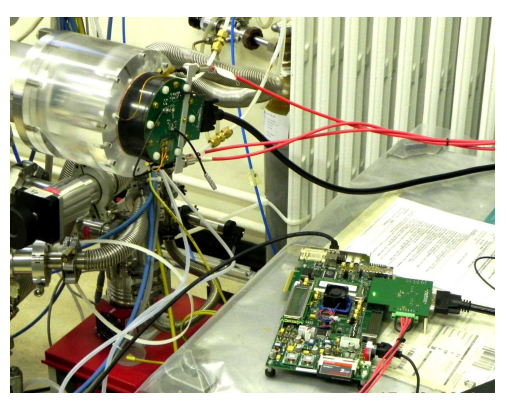

(b)

Figure 5: Pictures showing the X-ray generator and vacuum beamline (a) and the InGrid based $\mathrm{X}$-ray detector mounted on the beamline and its readout system (b).

Different combinations of target and filter materials were used together with adapted acceleration voltages in order to cover the whole energy range from a few hundred $\mathrm{eV}$ up to $8 \mathrm{keV}$. The length of the frames recorded with the Timepix ASIC were adjusted to minimize the rate of double events and empty events. As no trigger signal is available in the current setup, only the charge accumulated in each pixel could be measured. For a first analysis all fired-pixels of a frame are considered to originate from one X-ray photon. Cuts on geometrical properties like center position, eccentricity and width of the pixels distribution along the shortest axis were used to remove remaining double events and events where the electron cloud was only partially contained on the active area of the InGrid.

In figure 6 the resulting spectra obtained by simple pixel counting for the runs with aluminum and carbon target are shown. The resulting fluorescence lines for these targets are at $1486 \mathrm{eV}$ and $277 \mathrm{eV}$ respectively. This demonstrates that the InGrid based detector is able to detect photons from the carbon $\mathrm{K}_{\alpha}$ line at $277 \mathrm{eV}$.

\section{Installation of the detector at CAST}

In order to replace the pnCCD detector behind the meanwhile remounted X-ray telescope at the CAST experiment, a small vacuum system has been designed, built, and commissioned which allows to connect the InGrid based detector to the X-ray telescope. It also features a differential 


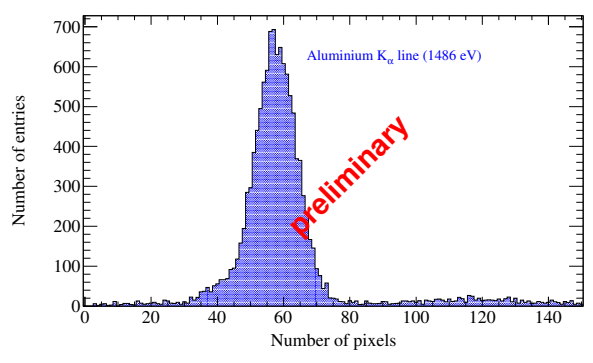

(a)

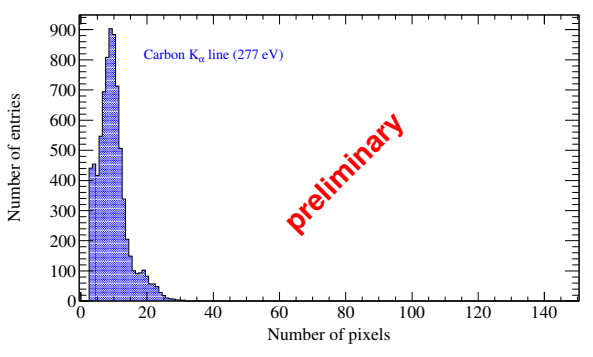

(b)

Figure 6: X-ray spectra obtained by pixel counting for the runs using an aluminum (a) and carbon (b) target respectively. Energies of the corresponding fluorescence lines are $1486 \mathrm{eV}$ for aluminum and $277 \mathrm{eV}$ for carbon.

pumping scheme to ensure the good vacuum required in the X-ray telescope. In April and May of 2014 the InGrid based X-ray detector has been installed along with all the infrastructure needed at the CAST experiment preparing for the 2014 run of the CAST experiment and the search for Axions and Chameleons. The detector installed behind the X-ray telescope at the CAST experiment is shown in figure 7.

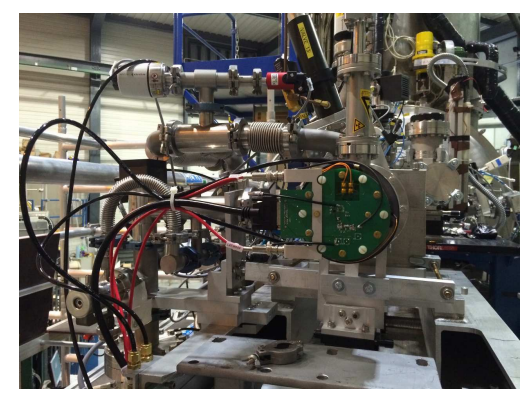

(a)

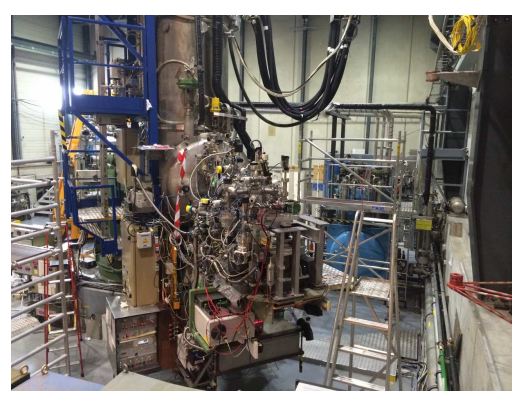

(b)

Figure 7: InGrid based X-ray detector mounted at the X-ray telescope of the CAST experiment along with all necessary infrastructure.

\section{Conclusion \& Outlook}

In order to provide CAST with a low energy X-ray detector with a low background rate, a detector based on the combination of a pixel chip and an integrated Micromegas stage has been developed. Following on the promising results with a first prototype a version of this InGrid based low energy X-ray detector which is suitable for the installation at CAST has been built. To demonstrate the detector's ability to detect low energy X-ray photons tests in the CAST detector lab were carried out. This facility provided an X-ray generator which can produce photons in the range of few hundred $\mathrm{eV}$ up to $8 \mathrm{keV}$. It could be successfully demonstrated that the InGrid based detector is able to detect photons from the carbon $\mathrm{K}_{\alpha}$ line at $277 \mathrm{eV}$. In April and May of 2014 the detector 
was installed along with its infrastructure at the CAST experiment behind the X-ray telescope and will take data during the 2014 run of the CAST experiment.

Still developments are on going especially concerning the readout of the induced mesh signal as well in the readout scheme as in the software and analysis tools. The addition of this should help to reduce the background rates further, it will be implemented as a future detector upgrade. Additionally, alternative materials for the X-ray window are investigated in order to reach higher transmissions especially in the sub $\mathrm{keV}$ range.

\section{References}

[1] P.A.R. Ade et al., Planck 2013 results. XVI. Cosmological parameters, astro-ph.CO/1303.5076

[2] C.A. Baker et al., Improved Experimental Limit on the Electric Dipole Moment of the Neutron, Phys. Rev. Lett. 97 (2006) 131801.

[3] R.D. Peccei and H.R. Quinn, CP Conservation in the Presence of Pseudoparticles, Phys. Rev. Lett. 38 (1977) 1440.

[4] S. Weinberg, A New Light Boson?, Phys. Rev. Lett. 40 (1978) 223.

[5] P. Brax et al., Detection prospects for solar and terrestrial chameleons, Phys. Rev. D 85 (2012) 043014 [hep-ph/1110.2583].

[6] K. Zioutas et al., A decommissioned LHC model magnet as an axion telescope, Nucl. Instr. Meth. Phys. Res. A 425 (1999) pp 480-487.

[7] M. Kuster et al., The x-ray telescope of CAST, New J. Phys. 9 (2007) 169 [phys ics / 0702188 ].

[8] M. Arik et al., Search for Solar Axions by the CERN Axion Solar Telescope with He3 Buffer Gas: Closing the Hot Dark Matter Gap, Phys. Rev. Lett. 112 (2014) 091302.

[9] M. Chefdeville et al., An electron-multiplying 'Micromegas' grid made in silicon wafer post-processing technology, Nucl. Instr. Meth. Phys. Res. A 556 (2006) pp 490-494.

[10] H. van der Graaf, GridPix: An integrated readout system for gaseous detectors with a pixel chip as anode, Nucl. Instr. Meth. Phys. Res. A 580 (2007) pp 1023-1026.

[11] X. Llopart et al., Timepix, a 65k programmable pixel readout chip for arrival time, energy and/or photon counting measurements, Nucl. Instr. Meth. Phys. Res. A 581 (2007) pp 485-494.

[12] Y. Bilevych et al., Spark protection layers for CMOS pixel anode chips in MPGDs, Nucl. Instr. Meth. Phys. Res. A 629 (2011) pp 66-73.

[13] M. Lupberger, The Pixel-TPC: first results from an 8-InGrid module, J. Instrum. 9 (2014) C01033.

[14] J. Galán et al., MICROMEGAS detectors in the CAST experiment, J. Instrum. 5 (2010) P01009.

[15] B.L. Henke et al., X-ray interactions: photoabsorption, scattering, transmission, and reflection at E=50-30000 eV, Z=1-92, At. Data Nucl. Data Tables 54 (1993) pp 181-342.

[16] C. Krieger et al., InGrid-based X-ray detector for low background searches, Nucl. Instr. Meth. Phys. Res. A 729 (2013) pp 905-909.

[17] S.J.C. do Carmo et al., Experimental Study of the w -Values and Fano Factors of Gaseous Xenon and Ar-Xe Mixtures for X-Rays, IEEE Trans. Nucl. Sci. 55-5 (2008) pp 2637-2642.

[18] T. Vafeiadis, Contribution to the search for solar axions in the CAST experiment, CERN, Aristotle University, Thessaloniki 2012 [CERN-THES IS-2012-349]. 